\title{
EXPERIMENTOS DIDÁTICOS UTILIZANDO SISTEMA DE ANÁLISE POR INJEÇÃo EM FLUXO
}

\author{
Fábio Rodrigo Piovezani Rocha \\ Departamento de Química - Universidade Federal de São Carlos - CP 676 - 13565-905 - São Carlos - SP \\ Patrícia Benedini Martelli e Boaventura Freire dos Reis \\ Centro de Energia Nuclear na Agricultura - Universidade de São Paulo - CP 96 - 13400-970 - Piracicaba - SP
}

Recebido em 23/9/98; aceito em 19/3/99

\begin{abstract}
DIDACTIC EXPERIMENTS EMPLOYING A FLOW INJECTION SYSTEM. Didactic experiments are proposed in order to demonstrate the characteristics of flow injection analysis and to extend the applications of FIA to the determination of physical chemistry parameters in undergraduate labs. All experiments can be performed with the same flow manifold by employing usual FIA devices. Analytical characteristics are presented by means of the determination of iron in river water, employing 1,10-phenantroline as chromogenic reagent. Physical chemistry applications were the determination of reaction stoichiometries by continuous variation and mole-ratio methods and the evaluation of the $\mathrm{pH}$ and ionic strength effects on the kinetic of the reduction of hexacianoferrate(III) by ascorbic acid.
\end{abstract}

Keywords: flow injection analysis; spectrophotometry; kinetics; stoichiometry.

\section{INTRODUÇÃO}

Sistemas de análise em fluxo, particularmente os sistemas de análise por injeção em fluxo (FIA), têm sido utilizados principalmente para a mecanização/automação de análises quími$\operatorname{cas}^{1,2}$. Empregando esses sistemas é possível a implementação de praticamente todas as etapas envolvidas no processo de análise química (amostragem, separações, diluições, pré-concentrações, adição de reagentes etc) $)^{1,2}$. Entretanto, embora pouco explorados nesse sentido, os sistemas também apresentam grande potencialidade para a determinação de parâmetros físico-químicos, tais como coeficientes de difusão ${ }^{3,4}$, viscosida$\mathrm{de}^{5}$, capacidade complexante de ligantes ${ }^{6,7}$, parâmetros cinéti$\cos ^{8,9}$ e estequiometria de reações ${ }^{10}$.

Embora extensamente utilizados em instituições de pesquisa e em análises de rotina ${ }^{11,12}$, discussões/experimentos sobre análise em fluxo são freqüentemente omitidos nos cursos de graduação em química. Apesar da importância do Brasil no desenvolvimento deste processo ${ }^{11}$, o número de trabalhos com enfoque didático em português é bastante restrito ${ }^{13,14}$.

Sistemas de análise por injeção em fluxo podem ser uma importante ferramenta em laboratórios de ensino de química. Além da demonstração dos fundamentos básicos de um importante e versátil processo analítico, sistemas FIA podem ser utilizados para ensinar/demonstrar conceitos importantes para a formação de profissionais na área de química. Experimentos clássicos podem ser implementados de forma mais atual, o que pode contribuir para o aumento do interesse dos alunos na execução de experimentos que poderiam ser considerados tediosos e repetitivos. Em vista disto, são propostos 3 experimentos que permitem apresentar o processo de análise por injeção em fluxo, através de aplicações úteis em laboratórios de ensino de Química Analítica e Físico-Química.

\section{SISTEMAS DE ANÁLISE POR INJEÇÃO EM FLUXO}

No processo de análise por injeção em fluxo, alíquotas de amostra (e eventualmente de reagentes) são inseridas em uma solução, que as transportada através do percurso analítico ${ }^{13}$. No decorrer do processo, a amostra sofre dispersão na solução transportadora, produzindo uma zona de amostra caracterizada pela existência de gradientes de concentração. Caso necessário, reações químicas podem ocorrer durante o transporte da zona de amostra em direção ao sistema de detecção. Em função da existência dos gradientes de concentração e da medida ser feita com a zona de amostra em movimento em relação ao sistema de detecção, obtém-se um sinal transiente, cuja altura pode ser relacionada à concentração inicial da espécie de interesse.

Para a implementação do processo FIA, é necessário um dispositivo para a propulsão dos fluidos, sendo mais frequientemente empregada a bomba peristáltica, que permite a propulsão a vazão constante. A seleção e a inserção de alíquotas pode ser feita empregando-se diversos dispositivos ${ }^{14}$, entre os quais destaca-se o injetor proporcional devido à simplicidade e versatilidade. Diversos detectores têm sido empregados em associação com o processo FIA ${ }^{1,2}$ e, em geral, são necessárias apenas pequenas modificações nos equipamentos convencionais. Completam o sistema, tubos de pequeno diâmetro (em geral 0,5 a $0,8 \mathrm{~mm}$ ), nos quais se processa a dispersão da amostra e ocorrem as reações químicas que, em geral, não se completam previamente à detecção.

O processo de dispersão da amostra, intrínseco aos sistemas de análise por injeção em fluxo, é dependente das características fisico-químicas das soluções (e.g. viscosidade), bem como das dimensões dos componentes do sistema (volume da alça de amostragem; material, diâmetro e comprimento dos tubos que constituem o percurso analítico). Usualmente, as medidas são efetuadas com esses parâmetros mantidos constantes, permitindo a obtenção de resultados caracterizados por alta repetibilidade. As vazões, volumes do percurso analítico e da amostra devem ser definidos considerando-se a dispersão e o tempo de residência necessário para a ocorrência das reações químicas.

\section{DESCRIÇÃO DOS EXPERIMENTOS}

Todos os experimentos podem ser executados utilizando um sistema FIA com zonas coalescentes ${ }^{15}$. Estes sistemas foram inicialmente propostos para reduzir o consumo de reagentes e, consequentemente, o custo das análises e a produção de resíduos tóxicos. Posteriormente, foram utilizados para efetuar neutralização de amostras com elevada acidez ${ }^{16}$, determinações simultâneas ${ }^{17}$, implementação da técnica de adições de padrão ${ }^{18}$ etc.

Com respeito à aplicação analítica, é proposta a determinação espectrofotométrica de ferro em águas empregando 1,10fenantrolina como reagente cromogênico. Este experimento é 
particularmente útil para a discussão de características analíticas importantes, tais como sensibilidade, seletividade, precisão e exatidão dos resultados. O segundo experimento consiste na determinação espectrofotométrica da estequiometria de compostos de coordenação, pelos métodos das variações contínuas (método de Job) e da razão molar (método de Yoe e Jones). Os procedimentos foram aplicados às reações entre cobre(II) e EDTA e ferro(II) e 1,10-fenantrolina. O terceiro experimento permite avaliar o efeito da acidez e da intensidade iônica sobre a cinética da reação entre hexacianoferrato(III) e ácido ascórbico.

\section{PARTE EXPERIMENTAL}

\section{Equipamentos e acessórios}

O módulo de análise foi construído utilizando um injetor proporcional $^{14,15}$ com duas seções construído em acrílico (Figura 1). Este dispositivo consiste de uma barra móvel, intercalada entre duas barras fixas. O movimento da barra central permite alternar entre os estágios de amostragem (Figura 1a) e de inserção de amostra (Figura 1b). Linhas de transmissão, alças de amostragem (Fig. 1, $\mathrm{L}_{1}$ e $\mathrm{L}_{2}$ ) e reatores helicoidais (Fig. 1, $\mathrm{B}_{1}$ e $\mathrm{B}_{2}$ ) foram feitos de tubos de polietileno, com diâmetro interno de $0,8 \mathrm{~mm}$. Para propulsão dos fluidos, foi utilizada uma bomba peristáltica com 8 canais (Ismatec, IPC8) e tubos de Tygon com diferentes diâmetros. Para a introdução dos reagentes por confluência foram utilizados conectores feitos de acrílico, na forma de "T". As medidas foram efetuadas utilizando um espectrofotômetro Femto, modelo 432, equipado com uma cela de fluxo de vidro ( $c a .180$ $\mu \mathrm{L})$ com caminho óptico de $10 \mathrm{~mm}$. Os sinais transientes foram monitorados através de um registrador potenciométrico (Cole-Parmer, modelo 60648).

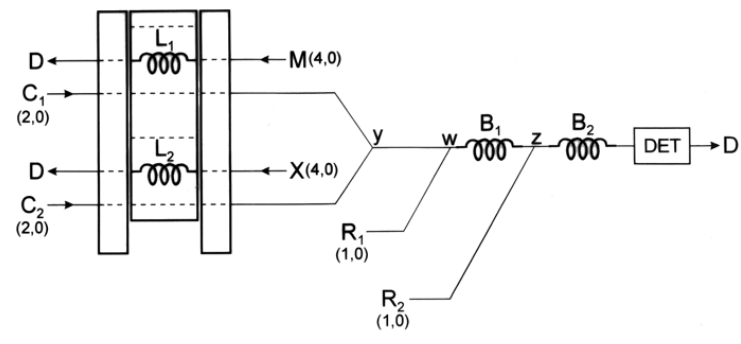

b

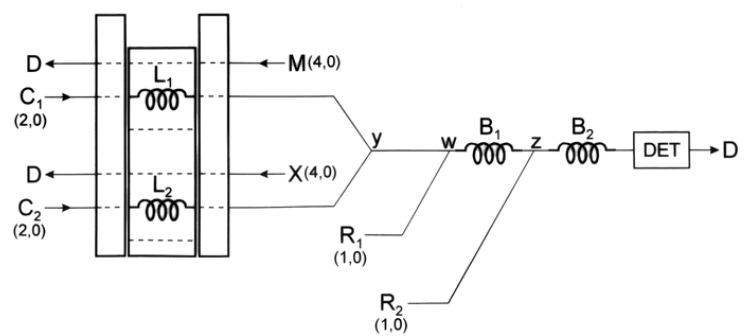

Figura 1. Diagrama de fluxos do sistema empregado em todos os experimentos. (a) posição de amostragem e (b) posição de inserção. $C_{1}, C_{2}$ - Fluxos transportadores; $R_{1}, R_{2}$ - reagentes adicionados por confluência. $L_{1}, L_{2}$ - alças de amostragem $(50 \mathrm{~cm} ; 250 \mu \mathrm{L}) . B_{1}, B_{2}$ reatores tubulares helicoidais (25 e $75 \mathrm{~cm}$, respectivamente). $y, w, z$ pontos de confluência. DET - espectrofotômetro. D - Descarte de soluções. Os números entre parênteses indicam vazões em $\mathrm{ml} / \mathrm{min}$.

\section{Reagentes e soluções}

Todas as soluções foram preparadas utilizando água destilada e deionizada e reagentes com grau de pureza analítico. As soluções empregadas em cada um dos experimentos são descritas a seguir:

\section{Determinação de ferro em águas}

Soluções de referência contendo 0,$5 ; 1,0 ; 2,0 ; 3,0$ e $4,0 \mathrm{mg}$ $\mathrm{L}^{-1} \mathrm{Fe}^{3+}$ foram preparadas em meio $0,014 \mathrm{~mol} \mathrm{~L}^{-1} \mathrm{HNO}_{3}$. Solução de $\mathrm{HNO}_{3}$ na mesma concentração foi empregada como transportador para as amostras e soluções de referência. Como reagente cromogênico foi utilizada solução $0,25 \%(\mathrm{~m} / \mathrm{v})$ de 1,10-fenantrolina, preparada por dissolução de quantidade apropriada do reagente em água quente $\left(60^{\circ} \mathrm{C}\right)$. Para redução de ferro(III), foi empregada solução $1,0 \%(\mathrm{~m} / \mathrm{v})$ de ácido ascórbico. $\mathrm{O}$ ajuste de acidez foi feito empregando-se solução tampão acetato $0,5 \mathrm{~mol} \mathrm{~L}^{-1}, \mathrm{pH} 4,7$.

\section{Determinação da estequiometria de compostos de coordenação}

Soluções separadas de ferro(III), em 0,014 mol L-1 $\mathrm{HNO}_{3}$, e de 1,10 -fenantrolina foram preparadas contendo $2,0 \times 10^{-5} ; 5,0$ x $10^{-5} ; 7,0 \times 10^{-5} ; 1,0 \times 10^{-4} ; 1,3 \times 10^{-4} ; 1,5 \times 10^{-4}, 1,8 \times 10^{-}$ 4 ou $2,0 \times 10^{-4} \mathrm{~mol} \mathrm{~L}^{-1}$. Tal como descrito no item anterior, soluções tampão acetato $0,5 \mathrm{~mol} \mathrm{~L}^{-1}(\mathrm{pH} \mathrm{4,7)} \mathrm{e} \mathrm{1,0 \%} \mathrm{(m/v)} \mathrm{de}$ ácido ascórbico foram empregadas para ajuste de acidez e para redução de ferro(III). Solução $0,014 \mathrm{~mol} \mathrm{~L}^{-1} \mathrm{HNO}_{3}$ foi empregada como transportador para as soluções de ferro(III).

Soluções de cobre(II) e $\mathrm{Na}_{2}$ EDTA foram preparadas separadamente contendo $5,0 \times 10^{-3}, 1,0 \times 10^{-2}, 1,5 \times 10^{-2}, 2,0 \times 10^{-2}$, $2,5 \times 10^{-2}, 3,0 \times 10^{-2}, 3,5 \times 10^{-2}$ ou $4,0 \times 10^{-2} \mathrm{~mol} \mathrm{~L}^{-1}$.

\section{Efeito da acidez e da intensidade iônica sobre a cinética da redução de hexacianoferrato(III) por ácido ascórbico}

Foram preparadas soluções $1,0 \times 10^{-3} \mathrm{~mol} \mathrm{~L}^{-1}$ de ácido ascórbico e hexacianoferrato(III) e soluções 0,$02 ; 0,05 ; 0,1$; 0,$2 ; 0,5$ e $1,0 \mathrm{~mol} \mathrm{~L}^{-1} \mathrm{NaNO}_{3}$. Para medidas em diferentes $\mathrm{pH}$, foram empregadas soluções tampão contendo: $0,06 \mathrm{~mol} \mathrm{~L}^{-1}$ tetraborato de sódio; $0,20 \mathrm{~mol} \mathrm{~L} \mathrm{~L}^{-1}$ hidrogenofosfato de sódio e $0,13 \mathrm{~mol} \mathrm{~L}^{-1}$ citrato de sódio, com $\mathrm{pH}$ ajustado para 2,0; 4,0; 6,$0 ; 8,0$ ou 9,1 com uma solução $5,0 \mathrm{~mol} \mathrm{~L}^{-1} \mathrm{HCl}$.

\section{Descrição do diagrama de fluxos}

O diagrama de fluxos do sistema com zonas coalescentes é mostrado na Figura 1. Na posição de amostragem (Fig. 1a), as alças de amostragem $L_{1}$ e $L_{2}$ são preenchidas pelas soluções representadas por $\mathrm{M}$ e $\mathrm{X}$. Quando a barra central do injetor é deslocada para a posição mostrada na Figura $1 \mathrm{~b}$, as alíquotas selecionadas das soluções $\mathrm{M}$ e $\mathrm{X}(250 \mu \mathrm{L})$ são simultaneamente inseridas nas soluções transportadoras $C_{1}$ e $C_{2}$, entrando em contato no ponto $y$. A zona de amostra resultante é transportada em direção a cela de fluxo do espectrofotômetro.

Quando necessário, os demais reagentes são introduzidos através dos fluxos $\mathrm{R}_{1}$ e $\mathrm{R}_{2}$, nos pontos de confluência $w$ e $z$. As reações químicas ocorrem nos reatores tubulares $B_{1}$ e $B_{2}$ (25 e $75 \mathrm{~cm}$, respectivamente). A detecção é feita com a amostra em movimento em relação ao detector e os sinais transientes são monitorados empregando um registrador potenciométrico.

\section{Procedimento}

\section{Determinação de ferro em águas}

A determinação de ferro foi feita empregando uma solução de 1,10-fenantrolina como reagente cromogênico (solução X), que foi introduzida através da alça de amostragem $L_{2}$. As amostras ou as soluções de referência de ferro (introduzidas através de $\mathrm{L}_{1}$ ) foram transportadas por uma solução $0,014 \mathrm{~mol} \mathrm{~L}^{-1} \mathrm{HNO}_{3}$ $\left(C_{1}\right)$. Água foi utilizada como transportador para o reagente $\left(C_{2}\right)$. As soluções de ácido ascórbico e tampão acetato foram introduzidas por confluência $\left(\mathrm{R}_{1}\right.$ e $\mathrm{R}_{2}$, respectivamente). As medidas foram efetuadas no comprimento de onda de $512 \mathrm{~nm}$. 
$\mathrm{O}$ procedimento foi aplicado à análise de 10 amostras de águas de rio. Para efeito de comparação, as mesmas amostras foram analisadas por espectrofotometria de absorção atômica com chama (FAAS). A repetibilidade dos resultados foi estimada através de 10 medidas para uma solução de ferro(III) contendo $2 \mathrm{mg} \mathrm{L}^{-1}$.

\section{Determinação da estequiometria de compostos de coordenação}

A estequiometria dos complexos formados entre cobre(II) e EDTA e ferro(II) e 1,10-fenantrolina (phen) foi determinada por dois métodos diferentes. O diagrama de fluxos mostrado na Figura 1 foi empregado com as soluções do íon metálico e do ligante sendo introduzidas como as soluções $\mathrm{M}$ e X, respectivamente. Apenas no caso da reação entre ferro(II) e phen foi necessária a introdução de reagentes por confluência: $\mathrm{R}_{1}-1 \%$ ácido ascórbico (utilizado para redução de ferro(III) a ferro(II)) e $\mathrm{R}_{2}$ solução tampão acetato (necessário para ajuste do $\mathrm{pH}$ para a reação de complexação). Como fluxo transportador das soluções de ferro, foi utilizada uma solução $0,014 \mathrm{~mol} \mathrm{~L}^{-1} \mathrm{HNO}_{3}$. As demais soluções foram transportadas por água. As medidas foram efetuadas nos comprimentos de onda de 745 e $512 \mathrm{~nm}$, para as reações entre $\mathrm{Cu}(\mathrm{II}) / \mathrm{EDTA}$ e $\mathrm{Fe}(\mathrm{II}) /$ phen, respectivamente.

$\mathrm{O}$ método das variações contínuas foi implementado mantendo-se constante a soma das concentrações do ligante $\left(C_{X}\right)$ e do íon metálico $\left(\mathrm{C}_{\mathrm{M}}\right)$, introduzidas nas alças de amostragem:

$\mathrm{C}_{\mathrm{Fe}}+\mathrm{C}_{\text {phen }}=2,0 \times 10^{-4} \mathrm{~mol} \mathrm{~L}^{-1} \mathrm{e}$

$\mathrm{C}_{\mathrm{Cu}}+\mathrm{C}_{\mathrm{EDTA}}=4,0 \times 10^{-2} \mathrm{~mol} \mathrm{~L}^{-1}$

No caso do método da razão molar, a concentração do íon metálico foi mantida constante $\left(5,0 \times 10^{-5} \mathrm{~mol} \mathrm{~L}^{-1} \mathrm{Fe}^{3+}\right.$ ou 2,5 x $10^{-2} \mathrm{~mol} \mathrm{~L}^{-1} \mathrm{Cu}^{2+}$ ), enquanto a concentração do ligante foi sistematicamente variada $\left(2,0 \times 10^{-5}-2,0 \times 10^{-4} \mathrm{~mol} \mathrm{~L}^{-1} \mathrm{Fe}^{3+}\right.$ ou $5,0 \times 10^{-3}-4,0 \times 10^{-2} \mathrm{~mol} \mathrm{~L}^{-1} \mathrm{Cu}^{2+}$ ).

A faixa de concentração de trabalho foi definida visando obter uma correlação linear entre os valores de absorbância e a concentração dos complexos. Isto foi verificado previamente para as soluções dos íons metálicos utilizando soluções contendo $0,003 \mathrm{~mol} \mathrm{~L}^{-1}$ phen ou $0,1 \mathrm{~mol} \mathrm{~L}^{-1} \mathrm{Na}_{2}$ EDTA. Na determinação da estequiometria pelo método das variações contínuas, os sinais de absorbância referentes ao complexo cobre(II)/ EDTA foram corrigidos, subtraindo o sinal correspondente ao íon cobre(II) nas diferentes concentrações. Estes valores foram obtidos substituindo-se a solução de $\mathrm{Na}_{2}$ EDTA por água.

\section{Efeito da acidez e da intensidade iônica sobre a cinética de redução do hexacianoferrato(III) por ácido ascórbico}

O diagrama de fluxos mostrado na Figura 1 foi empregado com a solução de ácido ascórbico sendo introduzida por confluência a $2,0 \mathrm{~mL} \min ^{-1}\left(\mathrm{R}_{1}\right)$. A alça de amostragem $\mathrm{L}_{1}$ foi preenchida pela solução de hexacianoferrato(III). Através da alça de amostragem $\mathrm{L}_{2}$, foram introduzidas as soluções tampão nos diferentes $\mathrm{pH}$ ou as soluções de nitrato de sódio nas diferentes concentrações. As medidas foram efetuadas em $418 \mathrm{~nm}$.

\section{RESULTADOS E DISCUSSÃO}

\section{Determinação de ferro em águas}

A aplicação mais usual dos sistemas de análise por injeção em fluxo é a determinação da concentração de espécies em solução. Neste sentido, sistemas FIA são empregados principalmente para a mecanização/automação de análises químicas, permitindo obter resultados caracterizados por elevadas repetibilidade e frequência analítica (número de determinações/ medidas que podem ser efetuadas por unidade de tempo), com baixo consumo de reagentes e amostras. Para demonstrar tais características, foi proposta a aplicação do sistema com zonas coalescentes para a determinação de ferro em amostras de águas de rio.

Uma característica intrínseca dos sistemas FIA é a dispersão controlada da amostra no fluxo transportador ${ }^{1,2,13}$. Em conseqüência, sinais transientes são gerados no detector, cuja altura é proporcional à concentração da espécie que está sendo medida. Uma vez que a vazão, o volume de amostra e o comprimento do percurso analítico são mantidos constantes, é possível a obtenção de sinais altamente reprodutíveis, apesar das medidas serem efetuadas sem que o estado de equilíbrio (químico e hidrodinâmico) tenha sido atingido. Por outro lado, a alteração do percurso analítico e principalmente do volume de amostra constitui uma maneira simples e rápida de ajustar o sinal obtido à faixa de resposta linear do instrumento de medida.

Os sinais transientes obtidos são mostrados na Figura 2. Uma vez que a lei de Beer é obedecida, as alturas dos sinais (valores de absorbância) são relacionadas com a concentração de ferro $\left(\mathrm{mg} \mathrm{L}^{-1}\right)$ nas soluções de referência através da equação: $\mathrm{A}=0,00025+0,08353 \mathrm{C}_{\mathrm{Fe}}(\mathrm{r}=0,9999)$, que permite estimar a concentração de ferro nas amostras. Um complemento útil a este experimento consiste em reduzir o volume de amostra, utilizando uma menor alça de amostragem, e verificar a redução de sensibilidade (coeficiente angular da curva analítica), devido ao aumento da dispersão da amostra.

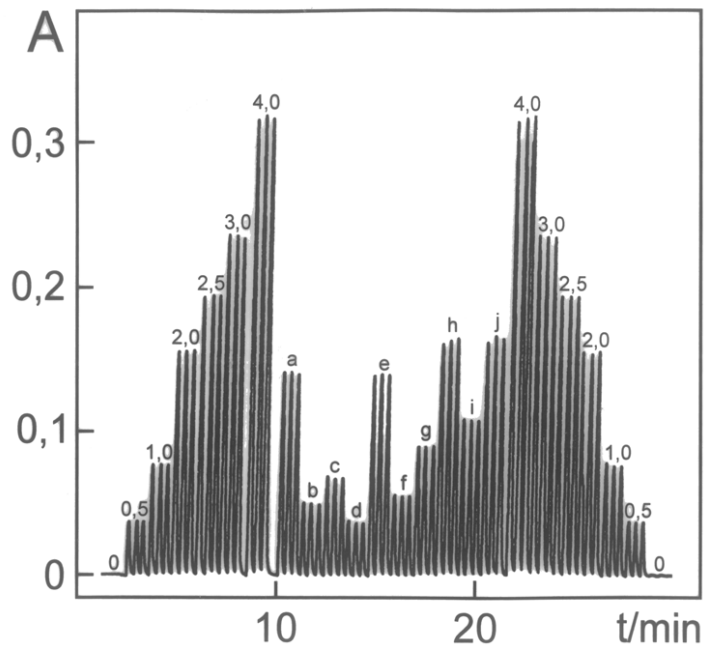

Figura 2. Registro dos sinais transientes para a determinação de ferro empregando 1,10-phenantrolina como reagente cromogênico. Números $\mathbf{0 - 4 , 0}$ indicam concentrações de ferro $\left(\mathrm{mg} \mathrm{L}^{-1}\right)$ nas soluções de referência e a-j - amostras de águas de rio. Medidas efetuadas em triplicata.

Os resultados obtidos para determinação de ferro em amostras de águas são mostrados na Tabela 1. Para efeito de comparação, são também mostrados os resultados obtidos por um procedimento de referência (espectrofotometria de absorção atômica com chama). Os resultados obtidos apresentam boa precisão (coeficiente de variação estimado em $0,6 \%, \mathrm{n}=10)$ e não são significativamente diferentes dos obtidos por FAAS ${ }^{19}$ a nível de confiança de $95 \%$. A discussão destes resultados com respeito à precisão e à exatidão é útil para demonstrar a importância destes parâmetros para um procedimento analítico.

Utilizando o diagrama de fluxos com zonas coalescentes (Fig. 1), o consumo de reagentes (e consequentemente o custo das análises e a produção de resíduos) é consideravelmente reduzido - 0,625 mg 1,10-phen / determinação. Para um sistema análogo com a introdução de reagente continuamente por confluência, o consumo foi estimado em 2,1 mg 1,10phen / determinação. Com o sistema apresentado, podem ser efetuadas $c a .140$ medidas/h, sendo o consumo de amostras igual a $250 \mu \mathrm{L}$ por determinação. 
Tabela 1. Determinação de ferro em amostras de água de rio.

\begin{tabular}{ccc}
\hline & \multicolumn{2}{c}{$\mathrm{C}_{\mathrm{Fe}} /\left(\mathrm{mg} \mathrm{L}^{-1}\right)^{*}$} \\
\hline Amostra & FIA & FAAS \\
\hline 1 & $1,17 \pm 0,01$ & $1,20 \pm 0,04$ \\
2 & $1,78 \pm 0,01$ & $1,61 \pm 0,02$ \\
3 & $2,12 \pm 0,02$ & $2,03 \pm 0,01$ \\
4 & $2,13 \pm 0,02$ & $2,05 \pm 0,02$ \\
5 & $1,41 \pm 0,01$ & $1,39 \pm 0.02$ \\
6 & $0,890 \pm 0,010$ & $0,901 \pm 0,021$ \\
7 & $0,660 \pm 0,010$ & $0,673 \pm 0,021$ \\
8 & $0,484 \pm 0,014$ & $0,444 \pm 0,036$ \\
9 & $0,719 \pm 0,007$ & $0,709 \pm 0,021$ \\
10 & $1,82 \pm 0,03$ & $1,75 \pm 0,02$
\end{tabular}

*medidas em triplicata

\section{Determinação da estequiometria de compostos de coordenação}

Utilizando um sistema com zonas coalescentes (Fig. 1) é possível implementar dois procedimentos clássicos para a determinação da estequiometria de reações através de medidas espectrofotométricas: método das variações contínuas e método da razão molar. Aspectos teóricos com respeito a estes métodos são bastante discutidos em textos com caráter didático ${ }^{10,20,21}$. Fundamentos básicos da análise por injeção em fluxo são adequadamente discutidos e experimentalmente demonstrados ${ }^{1,2,13}$.

Para a determinação da estequiometria, é necessário que a lei de Beer seja obedecida no intervalo de concentração em que as medidas espectrofotométricas são feitas ${ }^{20}$. Isto foi previamente verificado para as soluções dos íons metálicos empregando soluções contendo excesso dos ligantes. Na faixa de concentração em estudo, foram obtidas correlações lineares entre a absorbância e a concentração dos íons metálicos (mol $\mathrm{L}^{-1}$ ), representadas pelas equações:

$$
\begin{aligned}
& \mathrm{A}=-0,00399+5171 \mathrm{C}_{\mathrm{Fe}}, \mathrm{r}=0,9998 \\
& \mathrm{~A}=0.0219+19,56 \mathrm{C}_{\mathrm{Cu}}, \mathrm{r}=0,9992
\end{aligned}
$$

No método das variações contínuas, a soma das concentrações do íon metálico e do ligante é mantida constante, enquanto a proporção metal/ligante é sistematicamente variada. Isto foi obtido variando-se simultaneamente as concentrações das soluções M e X (Fig. 1), de modo que a soma se mantivesse constante. Após a inserção das soluções, as concentrações das espécies diminuem devido à diluição no ponto de confluência $y(1: 2)$ e ao processo de dispersão ${ }^{13}$. Entretanto, como a diluição/dispersão das alíquotas é comparável, em cada fração da zona de amostra a razão metal/ligante é mantida constante e igual a razão das concentrações das soluções inseridas nas soluções transportadoras $\left(\mathrm{C}_{1}\right.$ e $\left.\mathrm{C}_{2}\right)$. Entretanto, a dispersão ocorre de maneira diferenciada para cada fração da zona de amostra, formando um gradiente de concentração. Embora a soma das concentrações de M e X seja diferente para diferentes frações da zona de amostra, ela é mantida constante para um mesmo ponto (por exemplo, o correspondente ao sinal máximo). Desta forma, efetuando-se as medidas em um mesmo ponto é possível correlacionar os sinais obtidos com a fração molar (ou razão molar) de uma das espécies. Exceto quando mencionado, as medidas foram feitas no máximo do sinal transiente.

Os resultados obtidos para a reação entre ferro(II) e 1,10fenantrolina são mostrados na Figura 3a. Um gráfico dos valores de absorbância em função da fração molar de uma das espécies $\left(\mathrm{C}_{\mathrm{M}} /\left(\mathrm{C}_{\mathrm{M}}+\mathrm{C}_{\mathrm{X}}\right)\right.$ ou $\left.\mathrm{C}_{\mathrm{X}} /\left(\mathrm{C}_{\mathrm{M}}+\mathrm{C}_{\mathrm{X}}\right)\right)$ apresenta um máximo (ou mínimo, no caso da espécie absorvente ser consumida durante a reação) na fração molar correspondente à estequiometria do complexo (Fig. 4). Caso alguma das espécies (íon metálico ou ligante) absorva radiação no comprimento de onda de medida, o valor de absorbância deve ser corrigido levando-se em consideração a absorção da espécie em questão ${ }^{20}$. Isto foi necessário para a reação entre $\mathrm{Cu}^{2+} /$ EDTA, sendo a absorbância devida ao íon metálico subtraída da absorbância medida. Para as reações entre ferro(II)/phen e cobre(II)/EDTA, os valores máximos foram alcançados para $\mathrm{C}_{\mathrm{Fe}} /\left(\mathrm{C}_{\mathrm{Fe}}+\mathrm{C}_{\mathrm{phen}}\right)=0,25$ (Fig. 4, curva a) e $\mathrm{C}_{\mathrm{Cu}} / \mathrm{C}_{\mathrm{Cu}}+\mathrm{C}_{\mathrm{EDTA}}=0,5$ (Fig. 4 , curva b), indicando estequiometria 1:3 e 1:1 para os complexos:

$$
\begin{aligned}
& \mathrm{Fe}^{2+}+3 \text { phen } \rightleftharpoons\left[\mathrm{Fe}(\text { phen })_{3}\right]^{2+} \\
& \mathrm{Cu}^{2+}+\mathrm{EDTA}^{2-} \rightleftharpoons[\mathrm{Cu}(\mathrm{EDTA})]
\end{aligned}
$$

a

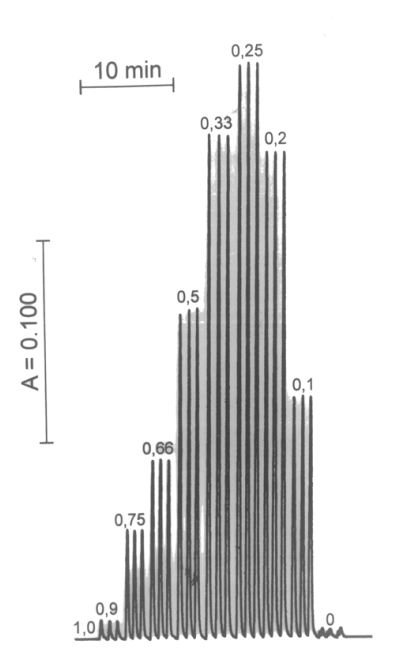

b

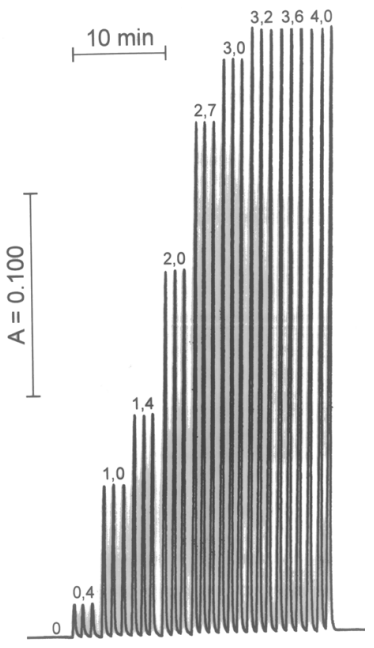

Figura 3. Registro dos sinais transientes obtidos para a determinação da estequiometria da reação entre ferro(II) e 1,10-fenantrolina. (a) Método das variações contínuas - números indicam a fração molar do ion metálico $\left(C_{\mathrm{Fe} 2+} / C_{\mathrm{Fe} 2+}+C_{p h e n}\right)$ e (b) método da razão molar - números representam a razão molar do ligante $\left(C_{p h e n} / C_{F e 2+}\right)$. Medidas efetuadas em triplicata.

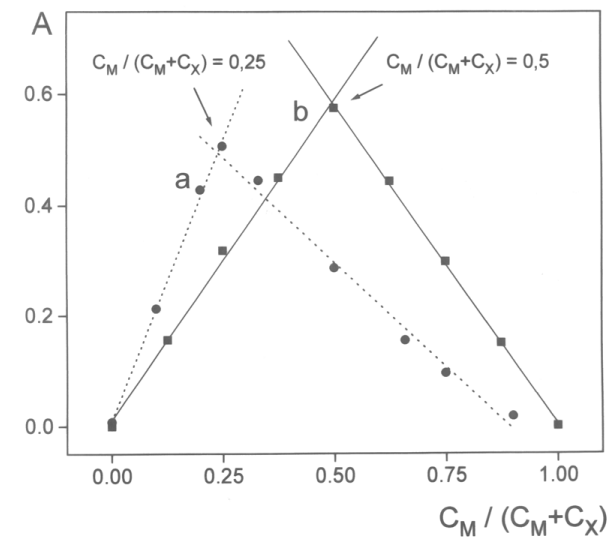

Figura 4. Gráficos de Job para a determinação da estequiometria dos complexos: (a) $\mathrm{Fe}^{2+} /$ phen $e$ (b) $\mathrm{Cu}^{2+} / E D T A$.

Uma das recomendações experimentais para a confirmação da estequiometria determinada pelo método de $\mathrm{Job}^{20}$ é repetir o experimento completo para diferentes valores da soma das concentrações do metal e do ligante. Quando o experimento é efetuado da maneira convencional, uma série de soluções contendo diferentes razões entre as concentrações do metal e do ligante é preparada, de forma a manter constante a soma das concentrações do metal e do ligante $\left(C_{M}+C_{X}=k_{1}\right)$. Para verificação, é recomendável repetir o experimento, mantendo-se 
a soma das concentrações constante, porém diferente daquela fixada no experimento inicial $\left(\mathrm{C}_{\mathrm{M}}+\mathrm{C}_{\mathrm{X}}=\mathrm{k}_{2} ; \mathrm{C}_{\mathrm{M}}+\mathrm{C}_{\mathrm{X}}=\mathrm{k}_{3}\right.$; com $\mathrm{k}_{1} \neq \mathrm{k}_{2} \neq \mathrm{k}_{3}$ ).

Os gradientes de concentração gerados devido à dispersão das soluções $\mathrm{M}$ e $\mathrm{X}$ podem ser explorados para confirmar a estequiometria dos complexos, efetuando-se medidas para diferentes valores da soma das concentrações de $\mathrm{M}$ e X (diferentes frações da zona de amostra). No caso do sistema empregado neste experimento (Fig. 1), cada fração da zona de amostra é caracterizada por uma concentração diferente de metal e de ligante, sendo a razão entre estas espécies mantida constante e

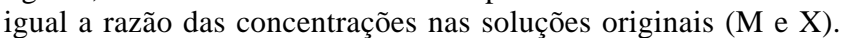

Na Figura 5a, são mostrados alguns sinais transientes obtidos para o complexo entre cobre(II) e EDTA, para diferentes frações molares do íon metálico. A soma das concentrações das soluções inseridas no fluxo transportador foi mantida constante $\left(\mathrm{C}_{\mathrm{Cu}}+\mathrm{C}_{\mathrm{EDTA}}=4,0 \times 10^{-2} \mathrm{~mol} \mathrm{~L}^{-1}\right)$, tal como para a obtenção dos resultados mostrados anteriormente (Figura 4). A partir destes resultados, foram efetuadas medidas em diferentes pontos dos sinais transientes para diferentes valores de $\mathrm{M}$ e $\mathrm{X}$ inseridas no fluxo transportador. Na prática, estas medidas foram feitas traçando-se uma linha perpendicular ao eixo x (Fig. 5a), de maneira a selecionar o mesmo ponto de medida para os diferentes sinais (o tempo decorrido após a inserção de $\mathrm{M}$ e $\mathrm{X}$ é mantido constante). $\mathrm{O}$ valores de absorbância são encontrados medindo-se a altura correspondente a cada um dos sinais, tomando-se a linha base como referência. Alguns valores encontrados são mostrados em função da fração molar do íon metálico na Figura 5b. Verificase que todas as curvas apresentam valor máximo em torno da fração molar $0,5\left(C_{M} /\left(C_{M}+C_{X}\right)=0,5\right)$, confirmando a estequiometria 1:1 para o complexo.
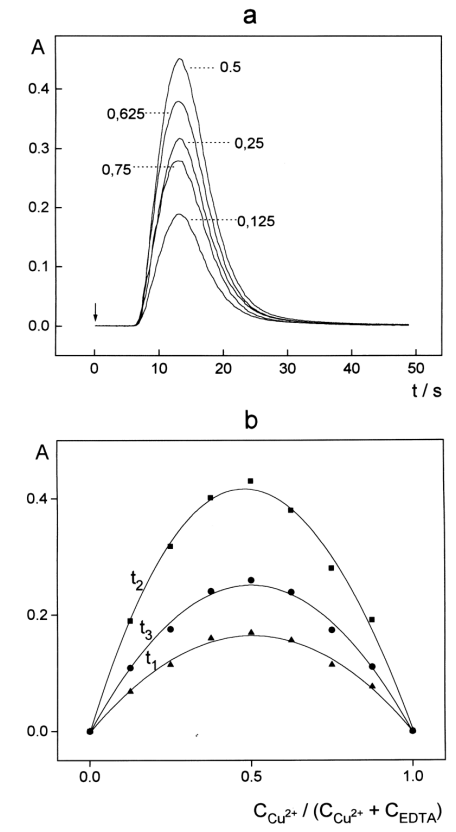

Figura 5. Determinação da estequiometria do complexo $\mathrm{Cu}^{2+} /$ EDTA explorando gradientes de concentração. (a) Sinais transientes para diferentes frações molares do íon metálico (instante de inserção da amostra indicado pela seta) e (b) Gráficos de Job obtidos através de medidas em diferentes tempos após a inserção da amostra: $t_{1}-9 \mathrm{~s}, t_{2}$ $-13 \mathrm{~s} e t_{3}-17 \mathrm{~s}$.

Para a aplicação do método da razão molar, a concentração de uma das espécies foi mantida constante, enquanto a concentração da outra foi sistematicamente variada. Na Figura 3b, são mostrados os resultados obtidos para a reação ferro(II)/phen, mantendo-se constante a concentração de ferro e variando-se a concentração do ligante. Se um gráfico de absorbância em função da razão molar de uma das espécies $\left(C_{M} / C_{X}\right.$ ou $\left.C_{X} / C_{M}\right)$ é construído, observa-se a variação de absorbância até a razão molar correspondente à estequiometria do complexo ser atingida. No caso do ligante absorver radiação no comprimento de onda de medida, verifica-se uma mudança de inclinação da curva após a razão estequiométrica ter sido alcançada. Neste caso, a intersecção das duas retas permite determinar a razão molar correspondente à estequiometria ${ }^{21}$. Para a reação entre ferro(II) e phen, a razão molar correspondente a estequiometria é alcançada para $\mathrm{C}_{\text {phen }} / \mathrm{C}_{\mathrm{Fe}}=3,2$ (Fig. 6, curva a), confirmando que 3 moléculas do ligante complexam cada íon metálico (eq.1). Para a reação entre cobre(II) e EDTA, observa-se variação de absorbância até $\mathrm{C}_{\mathrm{EDTA}} / \mathrm{C}_{\mathrm{Cu}}=1$ (Fig. 6, curva b), confirmando a estequiometria 1:1 para o complexo (eq.2). Tal como descrito para o método das variações contínuas, medidas em diferentes frações da zona de amostra (exploração dos gradientes de concentração) também podem ser utilizadas para confirmar a estequiometria do complexo. Diferentemente do que ocorre com o método de Job, não é necessário subtrair a absorbância devida ao íon cobre(II), visto que a concentração deste íon se mantém constante durante todo o experimento (note o sinal de absorbância correspondente a $C_{X} / C_{M}=0$; Fig. 6 , curva b).

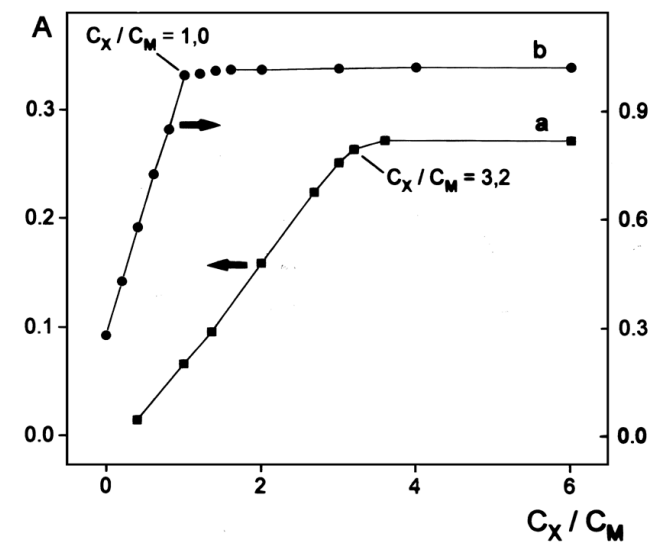

Figura 6. Determinação da estequiometria dos complexos pelo método da razão molar: (a) $\mathrm{Fe}^{2+} /$ phen e (b) $\mathrm{Cu}^{2+} / \mathrm{EDTA}$. As concentrações dos íons metálicos foram mantidas constantes: $C_{F e 2+}=5 \times 10^{-5} \mathrm{~mol} \mathrm{~L}^{-1}$ e $C_{\mathrm{Cu}_{2+}}=2,5 \times 10^{-2} \mathrm{~mol} \mathrm{L^{-1 }}$. As setas indicam as ordenadas para as curvas $\boldsymbol{a}$ e $\boldsymbol{b}$.

Efeito da acidez e da intensidade iônica sobre a cinética da redução do hexacianoferrato(III) pelo ácido ascórbico

O diagrama de fluxos mostrado na Figura 1 foi empregado para investigar qualitativamente o efeito da acidez $(\mathrm{pH})$ e da intensidade iônica sobre a cinética de redução do hexacianoferrato (III) pelo ácido ascórbico. Dentre as espécies envolvidas, apenas o ânion hexacianoferrato (III) absorve radiação no comprimento de onda de medida e a concentração remanescente após a reação é diretamente proporcional à absorbância para a faixa de concentração empregada.

O mecanismo da reação ${ }^{9,22}$ envolve a dissociação do ácido ascórbico $\left(\mathrm{H}_{2} \mathrm{~A}\right)$ para formar o ânion ascorbato $\mathrm{HA}^{-}$(etapa 1). $\mathrm{Na}$ segunda etapa, limitante da velocidade da reação, ocorre a transferência de um elétron do ânion ascorbato para o hexacianoferrato(III), gerando o radical livre ascorbato (HA $\left.{ }^{\bullet}\right)$. $\mathrm{Na}$ última etapa, este radical transfere um elétron para o hexacianoferrato(III):

De acordo com o mecanismo experimentalmente determinado, em valores constantes de $\mathrm{pH}$, a reação segue uma cinética de primeira ordem em relação a cada um dos reagentes (eq. $4)^{9,22}$. Adicionalmente, observou-se que a constante de velocidade da reação é inversamente proporcional a concentração de ácido presente no meio reacional (eq. 5) ${ }^{22}$ : 
$-\frac{1}{2} \frac{\mathrm{d}\left[\mathrm{Fe}(\mathrm{CN})_{6}^{3-}\right.}{\mathrm{dt}}=\mathrm{k}^{\prime}\left[\mathrm{Fe}(\mathrm{CN})_{6}^{3-}\right]\left[\mathrm{H}_{2} \mathrm{~A}\right]$

$\mathrm{k}^{\prime}=\mathrm{k} \frac{1}{\left[\mathrm{H}^{+}\right]}$

O efeito da acidez sobre a cinética da reação pode ser observado na Figura 7. Uma vez que o tempo de reação é mantido constante, os sinais de absorbância correspondentes aos diferentes valores de $\mathrm{pH}$ podem ser diretamente comparados. Verificase a diminuição exponencial da concentração remanescente de hexacianoferrato(III) com o aumento do $\mathrm{pH}$ do meio reacional. Isto ocorre devido à maior dissociação do ácido ascórbico (etapa 1), aumentando a concentração da espécie cineticamente ativa, o ânion ascorbato. Um experimento similar utilizando procedimentos convencionais seria dificultado pela necessidade de efetuar medidas em intervalos de tempo relativamente curtos, especialmente para valores mais altos de $\mathrm{pH}$.

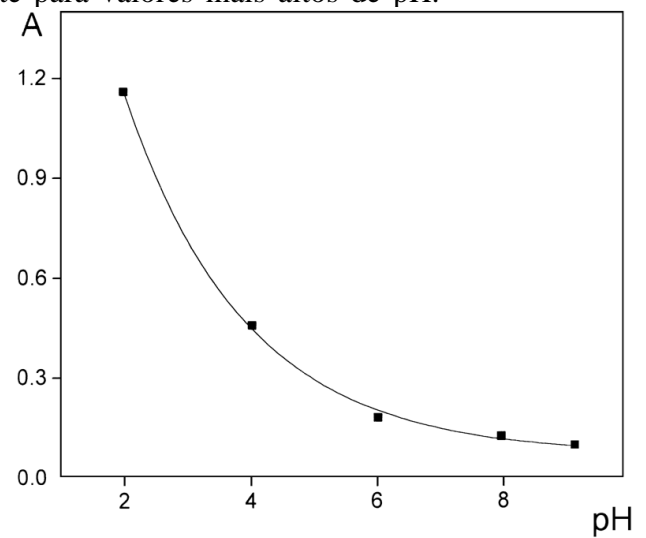

Figura 7. Efeito da acidez sobre a cinética de redução do hexacianoferrato(III) pelo ácido ascórbico.

O efeito da intensidade iônica sobre a cinética da redução é demonstrado na Figura 8. A etapa lenta da reação (etapa 2) envolve a colisão entre duas espécies aniônicas, que é dificultada do ponto de vista eletrostático. Desta forma, é esperado que a reação ocorra mais rapidamente quando a intensidade iônica do meio é aumentada. Isto ocorre devido à alteração da atmosfera iônica e, consequentemente, das densidades de carga ao redor dos íons ${ }^{9}$. Isto pode ser verificado na Figura 8, na qual o aumento da concentração de nitrato de sódio causa a diminuição da concentração remanescente de hexacianoferrato(III), indicando que a velocidade da reação aumenta a medida em que a intensidade iônica é aumentada.

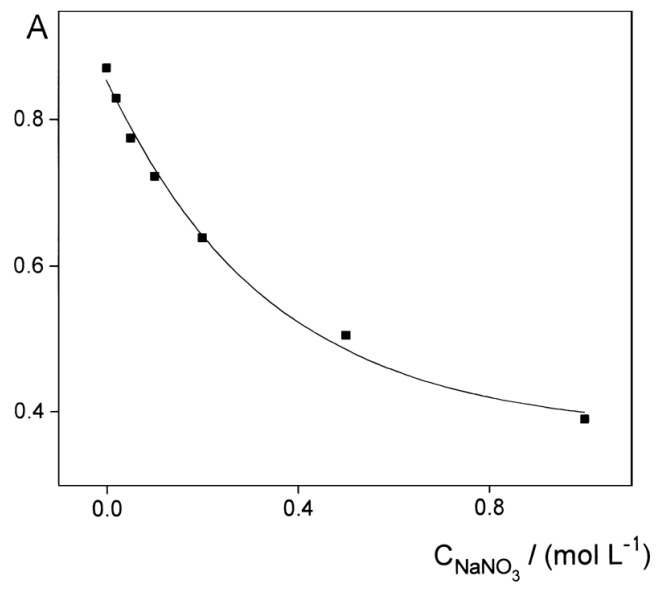

Figura 8. Efeito da intensidade iônica sobre a cinética de redução do hexacianoferrato(III) pelo ácido ascórbico.

\section{CONCLUSÕES}

A versatilidade dos sistemas de análise por injeção em fluxo foi demonstrada a partir de 3 experimentos que podem ser facilmente implementados em laboratórios de ensino de Química Analítica e Físico Química. Os experimentos podem ser executados empregando praticamente o mesmo módulo de análise, construído utilizando componentes usuais em análise por injeção em fluxo. Através deles, é possível apresentar um processo cinético de análise, discutindo sobre seus fundamentos e demonstrando algumas de suas características, tais como alta repetibilidade dos resultados, baixo consumo de reagentes e elevada frequência analítica.

Dois procedimentos clássicos para a determinação da estequiometria de reações químicas podem ser implementados. O procedimento é empregado para a determinação da estequiometria de dois compostos de coordenação. $\mathrm{O}$ emprego do sistema com zonas coalescentes permite reduzir o número de soluções a serem preparadas, além de tornar os procedimentos mais rápidos e menos tediosos. Devido às características dos sistemas FIA, é possível a determinação da estequiometria de reações que envolvam a formação de produtos instáveis ou insolúveis. A estequiometria de reações com cinética lenta pode ser determinada sem a necessidade de que o estado de equilíbrio químico seja alcançado, devido à temporização reprodutível característica dos sistemas FIA. Adicionalmente, podem ser demonstradas as potencialidades da exploração dos gradientes de concentração gerados devido ao processo de dispersão.

Devido à característica dinâmica dos sistemas em fluxo, foi possível estimar o efeito da acidez e da intensidade iônica sobre a cinética de uma reação química. $\mathrm{Na}$ maioria dos casos, esta informação qualitativa é suficiente para demonstrar o efeito de diversos fatores sobre a cinética da reação, evitando a necessidade de empregar procedimentos mais complexos para a determinação de constantes de velocidade, por exemplo. A execução de experimentos similares por procedimentos convencionais é dificultada pela necessidade do controle manual do tempo decorrido desde a mistura dos reagentes até a medida dos sinais. Neste caso, o acompanhamento de reações com cinética rápida torna-se difícil e erros podem surgir devido ao controle não rigoroso do tempo de reação.

\section{AGRADECIMENTOS}

Os autores agradecem a I. L. Mattos pelas sugestões apresentadas e a FAPESP, CNPq e CAPES pelo apoio financeiro.

\section{REFERÊNCIAS}

1. Ruzicka, J.; Hansen, E. H.; Flow Injection Analysis; $2^{\underline{a}}$ ed., John Wiley \& sons; New York, 1988.

2. Karlberg, B.; Pacey, G. E.; Flow Injection Analysis. A Practical Guide; Elsevier; Amsterdam, 1989.

3. Adams, R.N.; Gerhardt, G.; Anal. Chem. 1982, 54, 2618.

4. Vanderslice, J.T.; Beecher, G.R.; Rosenfeld, A.G.; Anal. Chem. 1984, 56, 292.

5. Betteridge, D.; Cheng, W.C.; Dagless, E.L.; David, P.; Goad, T.B.; Deans, D.R.; Newton, D.A.; Pierce, T.B.; Analyst 1983, 108, 1.

6. Yoza, N.; Miyaji, T.; Hirai, Y.; Ohashi, S.; J. Chromatogr. 1984, 283, 89.

7. Yoza, N.; Shuto, T.; Baba, Y.; Tanaka, A.; Ohashi, S.; J. Chromatogr. 1984, 298, 419.

8. Hungerford, J. M.; Christian, G. D.; Ruzicka, J.; Giddings, J. C.; Anal. Chem. 1985, 57, 1794.

9. Nóbrega, J.A.; Rocha, F.R.P.; J. Chem. Educ. 1997, $74,560$.

10. Rios, A.; Luque de Castro, M.D.; Valcárcel, M.; J. Chem Educ. 1986, 63, 552. 
11. Reis, B.F.; Quím. Nova 1996, 19, 51.

12. Nóbrega, J. A., Neves, E. F. A., Rocha, F. R. P., Oliveira, P. V.; Luccas, P. O.; Gouveia, S. T.; Quím. Nova 1996, 19,684

13. Reis, B.F.; Giné, M. F.; Kronka, E. A. M.; Quím. Nova 1989, $12,82$.

14. Reis, B.F.; Bergamin $\mathrm{F}^{\mathrm{o}}$, H.; Quím. Nova 1993, 16, 570.

15. Bergamin $\mathrm{F}^{\mathrm{o}}$, H.; Zagatto, E. A. G.; Krug, F. J.; Reis, B. F.; Anal. Chim. Acta 1978, 101, 17.

16. Reis, B. F.; Bergamin Fo , H.; Zagatto, E. A. G.; Krug, F. J.; Anal. Chim. Acta 1979, 107, 309.
17. Reis, B. F.; Zagatto, E. A. G.; Jacintho, A. O.; Krug, F. J.; Bergamin $\mathrm{F}^{\mathrm{o}}$, H.; Anal. Chim. Acta 1980, 119, 305.

18. Giné, M.F.; Reis, B.F.; Zagatto, E.A.G.; Krug, F.J.; Jacintho, A.O.; Anal. Chim. Acta 1983, 155, 131.

19. Harris, D. C.; Quantitative Chemical Analysis; 4th ed.; W.H. Freeman and Company; New York, 1995; p. 62-69.

20. Hill, Z. D.; MacCarthy, P.; J. Chem. Educ. 1986, 63, 162.

21. Skoog, D. A.; West, D. M.; Holler, F. J.; Fundamentals of Analytical Chemistry; $6^{\text {th }}$ ed.; Saunders, Philadelphia, 1992; p.582.

22. Martins, L. J. A.; Costa, J. B.; J. Chem. Educ. 1988, 65, 176. 